\title{
Palladium-Catalyzed Aminoacetoxylation of Alkenes
}

\author{
Erik J. Alexanian, Chulbom Lee, and Erik J. Sorensen* \\ Department of Chemistry, Princeton University, Princeton, NJ 08544
}

\section{Supporting Information}

General: All reactions were carried out under an atmosphere of Ar unless otherwise indicated. Acetonitrile and dichloromethane were dried by passing through activated alumina columns. Palladium(II) acetate and bis(benzonitrile)palladium(II) chloride was purchased from Strem Chemical, and iodobenzene diacetate was purchased from Acros Organics (both used as received). Tetrabutylammonium acetate (Aldrich) is hygroscopic, and was stored in a glovebox. Other commercial reagents of high purity were purchased and used without further purification, unless otherwise noted. Reactions were monitored by thin-layer chromatography (TLC) carried out on $0.25 \mathrm{~mm}$ Merck silica gel plates (60 $\mathrm{F}_{254}$ ) using UV light as a visualizing agent and aqueous ceric sulfate/phosphomolybdic acid, aqueous potassium permanganate, or ethanolic $p$-anisaldehyde solution and heat as developing agents. E. Merck silica gel 60 (230-400 mesh) was used for flash column chromatography.

Instrumentation: FT-IR spectra were obtained on a Perkin-Elmer Paragon 500. Nuclear magnetic resonance (NMR) spectra were taken on a Varian Inova-500, Inova-400, and Mercury-Vx-300 instruments. Proton chemical shifts are reported in parts per million downfield from tetramethylsilane and are calibrated to the residual protium $(7.26 \mathrm{ppm})$ in the $\mathrm{CDCl}_{3} \mathrm{NMR}$ solvent. Carbon chemical shifts are reported in parts per million downfield from tetramethylsilane and are calibrated to the solvent peaks (77 ppm). $d^{6}$ DMSO chemical shifts are reported in the same manner, calibrated to the solvent peaks $\left({ }^{1} \mathrm{H}=2.49 \mathrm{ppm},{ }^{13} \mathrm{C}=39.5 \mathrm{ppm}\right)$. Coupling constant values were extracted assuming first-order coupling. The multiplicities are abbreviated as follows: $\mathrm{s}=$ singlet, $\mathrm{d}=$ doublet, $\mathrm{t}=$ triplet, $\mathrm{q}=$ quartet, $\mathrm{m}=$ multiplet, app $\mathrm{s}=$ apparent singlet, app $\mathrm{d}=$ apparent doublet, app $\mathrm{dt}=$ apparent doublet of triplets, app $\mathrm{t}=$ apparent triplet, app $\mathrm{q}=$ apparent quartet, br = broad signal. High resolution mass spectra were obtained using an Agilent ESI-TOF mass spectrometer.

Experimental: All carbamate substrates were prepared by simple condensation of the appropriate alcohol with $p$-toluenesulfonyl isocyanate (Aldrich). Primary $\mathrm{N}$ nosylsulfonamides were prepared in one step from the corresponding alcohol under Mitsunobu reaction conditions. ${ }^{1}$ Secondary $N$-nosylsulfonamide substrate (Table 1 , entry 6) was prepared in two steps from the corresponding cyclopentanol via Mitsunobu reaction/TFA deprotection steps. ${ }^{2} N$-Tosylamide substrate (entry 2) was prepared as reported by Feringa. ${ }^{3} 2$-Allyl $N$-tosylanilide substrate (entry 5 ) was prepared as reported by Larock. ${ }^{4}$ 


\section{Representative Procedures for the Intramolecular Aminoacetoxylation of Alkenes}

Condition A (Synthesis of 13): Palladium(II) acetate $(4.7 \mathrm{mg}, 0.021 \mathrm{mmol})$ and iodobenzene diacetate $(136.0 \mathrm{mg}, 0.42 \mathrm{mmol})$ were placed in a one-dram screw-cap vial with Teflon cap containing a Teflon-coated stirbar. Tetrabutylammonium acetate (63.7 $\mathrm{mg}, 0.21 \mathrm{mmol}$ ) was placed in a one-dram screw-cap vial with Teflon cap and was removed from the glovebox. The tetrabutylammonium acetate was dissolved in $550 \mu \mathrm{l}$ $\mathrm{CH}_{2} \mathrm{Cl}_{2}$ and added to the vial containing the catalyst and oxidant and stirred under Ar. The substrate $N$-nosylsulfonamide $(60.1 \mathrm{mg}, 0.21 \mathrm{mmol})$ was dissolved in $550 \mu \mathrm{CH}_{2} \mathrm{Cl}_{2}$ and added dropwise to the reaction vial. The reaction was stirred at $25^{\circ} \mathrm{C}$ until completion (5 h). The reaction was diluted with $\mathrm{CH}_{2} \mathrm{Cl}_{2}(1 \mathrm{~mL})$ and washed with water $(1 \mathrm{~mL})$. The resulting organic extract was washed with brine $(1 \mathrm{~mL})$, separated, and the brine layer was extracted with $\mathrm{CH}_{2} \mathrm{Cl}_{2}(1 \mathrm{~mL})$. The combined organics were dried $\left(\mathrm{MgSO}_{4}\right)$ and concentrated by rotary evaporation. Flash chromatography $\left(8: 8: 1\right.$ hexanes $\left./ \mathrm{CH}_{2} \mathrm{Cl}_{2} / \mathrm{Et}_{2} \mathrm{O}\right)$ yielded $\mathbf{1 3}$ (57.9 $\mathrm{mg}, 80 \%)$.

Condition B (Synthesis of 8): Bis(benzonitrile)palladium(II) chloride (19.2 mg, 0.050 $\mathrm{mmol})$ and iodobenzene diacetate $(644.2 \mathrm{mg}, 2.00 \mathrm{mmol})$ were placed in a six-dram screw-cap vial with rubber septa containing a Teflon-coated stirbar, and dissolved in 2.5 $\mathrm{mL}$ of $\mathrm{CH}_{2} \mathrm{Cl}_{2}$. The substrate $\mathrm{N}$-tosyl carbamate $(269.7 \mathrm{mg}, 1.00 \mathrm{mmol})$ was dissolved in $2.5 \mathrm{~mL}$ of $\mathrm{CH}_{2} \mathrm{Cl}_{2}$ and added to the reaction mixture dropwise. The reaction was stirred at $25{ }^{\circ} \mathrm{C}$ until complete $(12 \mathrm{~h})$ under Ar. The reaction was diluted with $\mathrm{CH}_{2} \mathrm{Cl}_{2}(5 \mathrm{~mL})$ and washed with water $(5 \mathrm{~mL})$. The resulting organic extract was washed with brine $(5 \mathrm{~mL})$, separated, and the brine layer was extracted with $\mathrm{CH}_{2} \mathrm{Cl}_{2}(5 \mathrm{~mL})$. The combined organics were dried $\left(\mathrm{MgSO}_{4}\right)$ and concentrated by rotary evaporation. Flash chromatography $\left(4: 4: 1 \rightarrow\right.$ 2:2:1 hexanes $\left./ \mathrm{CH}_{2} \mathrm{Cl}_{2} / \mathrm{Et}_{2} \mathrm{O}\right)$ yielded $\mathbf{8}(182.4 \mathrm{mg}, 56 \%)$.

Condition C (Synthesis of $\mathbf{2}$ and $\mathbf{3}$ ): Palladium(II) acetate $(4.8 \mathrm{mg}, 0.021 \mathrm{mmol})$ and iodobenzene diacetate $(137.2 \mathrm{mg}, 0.43 \mathrm{mmol})$ were placed in a one-dram screw-cap vial with Teflon cap containing a Teflon-coated stirbar, and dissolved in $550 \mu \mathrm{l}$ of 1:1 $\mathrm{AcOH}: \mathrm{Ac}_{2} \mathrm{O}$. The substrate $N$-nosylamide $(57.6 \mathrm{mg}, 0.21 \mathrm{mmol}$ ) was dissolved in $550 \mu \mathrm{l}$ of $1: 1 \mathrm{AcOH}: \mathrm{Ac}_{2} \mathrm{O}$ and added to the reaction mixture dropwise. The reaction was stirred at $25{ }^{\circ} \mathrm{C}$ until complete $(12 \mathrm{~h})$ under Ar. The reaction was dissolved in $5 \mathrm{ml} \mathrm{CH}_{2} \mathrm{Cl}_{2}$ and washed with water $(3 \mathrm{~mL})$. The resulting organic extract was washed with brine $(3 \mathrm{~mL})$, separated, and the brine layer was extracted with $\mathrm{CH}_{2} \mathrm{Cl}_{2}(5 \mathrm{~mL})$. The combined organics were dried $\left(\mathrm{MgSO}_{4}\right)$ and concentrated by rotary evaporation. Flash chromatography (2:2:1 hexanes $/ \mathrm{CH}_{2} \mathrm{Cl}_{2} / \mathrm{Et}_{2} \mathrm{O}$ ) yielded $\mathbf{2}$ and $\mathbf{3}$ as a 9:1 mixture of inseparable regioisomers $(61.0 \mathrm{mg}, 87 \%)$. The reaction conducted under air atmosphere gave a similar yield $(88 \%)$.

\section{Control Experiments}

Control experiments were run using a variety of nitrogen nucleophiles and all three conditions to determine the extent of background reaction in the absence of palladium. The experiments were performed by simply omitting the palladium catalysts from the standard protocols as described above. No significant reaction occurred in the absence of palladium using conditions A or B; the $N$-tosyl amide (Table 1, entry 2), allyl alcohol- 
derived carbamate (entry 3), and 1,1-disubstituted nosylamide (entry 7) were all studied. Condition $\mathrm{C}$ (studied using compound $\mathbf{1}$ as substrate), however, did facilitate a slow background reaction in the absence of palladium.

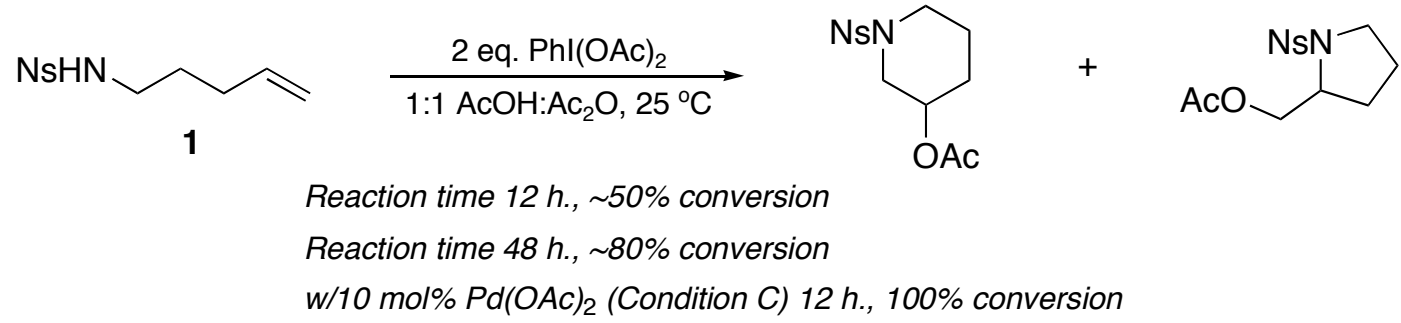

Interestingly, it appears that although slow, the regioselectivity of this background reaction compares well with that in the presence of palladium ( 9:1 endo:exo cyclization by ${ }^{1} \mathrm{H}$ NMR). Further studies to explore the nature of this reaction are underway. ${ }^{5}$

\section{Physical Data for Aminoacetoxylation Products:}

2:<smiles>CC(=O)OC1CCCN(S(=O)(=O)[O-])C1</smiles>

Purified by flash chromatography $\left(2: 2: 1\right.$ hexanes $\left./ \mathrm{CH}_{2} \mathrm{Cl}_{2} / \mathrm{Et}_{2} \mathrm{O}\right)$, inseparable from minor regioisomer 3; TLC: $\mathrm{R}_{f}=0.49$ (3:3:1 hexanes $/ \mathrm{CH}_{2} \mathrm{Cl}_{2} / \mathrm{Et}_{2} \mathrm{O}$ ); IR (film) 1736, 1545, 1374, 1242, $1168 \mathrm{~cm}^{-1} ;{ }^{1} \mathrm{H}$ NMR $\left(500 \mathrm{MHz}, \mathrm{CDCl}_{3}\right) \delta 7.99(\mathrm{~m}, 1 \mathrm{H}), 7.69(\mathrm{~m}, 2 \mathrm{H}), 7.62(\mathrm{~m}$, $1 \mathrm{H}), 4.83(\mathrm{~m}, 1 \mathrm{H}), 3.34-3.41(\mathrm{~m}, 3 \mathrm{H}), 3.24(\mathrm{~m}, 1 \mathrm{H}), 2.01(\mathrm{~s}, 3 \mathrm{H}), 1.87-1.98(\mathrm{~m}, 1 \mathrm{H})$, $1.77-1.85(\mathrm{~m}, 1 \mathrm{H}), 1.59-1.73(\mathrm{~m}, 2 \mathrm{H}) ;{ }^{13} \mathrm{C}$ NMR $\left(125 \mathrm{MHz}, \mathrm{CDCl}_{3}\right) \delta 170.2,148.0$, 133.6, 132.3, 131.6, 130.9, 124.1, 67.0, 48.6, 45.9, 28.3, 21.7, 21.0; HRMS (ESI-TOF) calculated for $[\mathrm{M}+\mathrm{Na}]^{+} 351.0621$, found 351.0625 .

4:

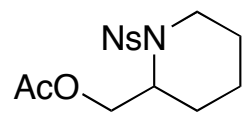

Purified by flash chromatography (8:8:1 hexanes/ $\left.\mathrm{CH}_{2} \mathrm{Cl}_{2} / \mathrm{Et}_{2} \mathrm{O}\right)$, separable from regioisomer 5 via preparative $\mathrm{TLC}\left(2: 1 \quad \mathrm{Et}_{2} \mathrm{O} /\right.$ pentane); TLC: $\mathrm{R}_{f}=0.55$ (2:2:1 hexanes $/ \mathrm{CH}_{2} \mathrm{Cl}_{2} / \mathrm{Et}_{2} \mathrm{O}$ ); IR (film) 1736, 1545, 1368, 1233, $1165 \mathrm{~cm}^{-1} ;{ }^{1} \mathrm{H}$ NMR (500 MHz, $\left.\mathrm{CDCl}_{3}\right) \delta 8.10(\mathrm{~m}, 1 \mathrm{H}), 7.68(\mathrm{~m}, 3 \mathrm{H}), 4.38(\mathrm{dd}, J=11.3,2.1 \mathrm{~Hz}, 1 \mathrm{H}), 4.32(\mathrm{~m}, 1 \mathrm{H}), 4.10$ (app q, $J=5.5 \mathrm{~Hz}, 1 \mathrm{H}), 3.66(\operatorname{app~d}, J=14 \mathrm{~Hz}, 1 \mathrm{H}), 3.13(\operatorname{app~t}, J=13.5 \mathrm{~Hz}, 1 \mathrm{H}), 1.95$ $(\mathrm{s}, 3 \mathrm{H}), 1.78(\mathrm{~m}, 1 \mathrm{H}), 1.57-1.73(\mathrm{~m}, 2 \mathrm{H}), 1.51(\mathrm{~m}, 1 \mathrm{H}), 1.39(\mathrm{~m}, 2 \mathrm{H}) ;{ }^{13} \mathrm{C}$ NMR $(125$ $\left.\mathrm{MHz} \mathrm{CDCl}_{3}\right) \delta 170.8,147.7,134.2,133.3,131.8,131.2,124.4,61.0,51.7,41.5,29.7$, 25.3, 25.0, 20.8; HRMS (ESI-TOF) calculated for $[\mathrm{M}+\mathrm{Na}]^{+} 365.0778$, found 365.0775.

5:

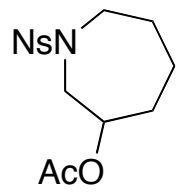


Purified by flash chromatography (8:8:1 hexanes/ $\left.\mathrm{CH}_{2} \mathrm{Cl}_{2} / \mathrm{Et}_{2} \mathrm{O}\right)$, separable from regioisomer 4 via preparative $\mathrm{TLC}\left(2: 1 \quad \mathrm{Et}_{2} \mathrm{O} /\right.$ pentane); TLC: $\mathrm{R}_{f}=0.54$ (2:2:1 hexanes $/ \mathrm{CH}_{2} \mathrm{Cl}_{2} / \mathrm{Et}_{2} \mathrm{O}$ ); IR (film) 1734, 1544, 1348, 1244, $1162 \mathrm{~cm}^{-1} ;{ }^{1} \mathrm{H}$ NMR $(500 \mathrm{MHz}$, $\left.\mathrm{CDCl}_{3}\right) \delta 8.01(\mathrm{~m}, 1 \mathrm{H}), 7.68(\mathrm{~m}, 2 \mathrm{H}), 7.62(\mathrm{~m}, 1 \mathrm{H}), 5.05(\mathrm{~m}, 1 \mathrm{H}), 3.58(\mathrm{dd}, J=15.3,4.6$ $\mathrm{Hz}, 1 \mathrm{H}), 3.47(\mathrm{dd}, J=15.0,6.1 \mathrm{~Hz}, 1 \mathrm{H}), 3.38(\mathrm{~m}, 2 \mathrm{H}), 2.05(\mathrm{~s}, 3 \mathrm{H}), 1.89-1.94(\mathrm{~m}, 1 \mathrm{H})$, 1.73-1.89 (m, 3H), 1.55-1.66 (m, 2H); $\left.{ }^{13} \mathrm{C} \mathrm{NMR} \mathrm{(125} \mathrm{MHz,} \mathrm{CDCl}_{3}\right) \delta 170.3,148.0$, 133.4, 132.9, 131.6, 131.1, 124.1, 71.9, 51.6, 49.9, 32.0, 29.7, 29.3, 21.1; HRMS (ESITOF) calculated for $[\mathrm{M}+\mathrm{Na}]^{+} 365.0778$, found 365.0780 .

6:

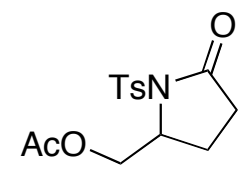

Purified by flash chromatography $\left(6: 6: 1\right.$ hexanes/ $\left.\mathrm{CH}_{2} \mathrm{Cl}_{2} / \mathrm{Et}_{2} \mathrm{O}\right) ;$ TLC: $\mathrm{R}_{f}=0.37(2: 2: 1$ hexanes $/ \mathrm{CH}_{2} \mathrm{Cl}_{2} / \mathrm{Et}_{2} \mathrm{O}$ ); IR (film) 1741, 1357, 1232, $1168 \mathrm{~cm}^{-1} ;{ }^{1} \mathrm{H}$ NMR (500 MHz, $\left.\mathrm{CDCl}_{3}\right) \delta 7.93(\mathrm{~d}, J=8.5 \mathrm{~Hz}, 2 \mathrm{H}), 7.32(\mathrm{~d}, J=8.0 \mathrm{~Hz}, 2 \mathrm{H}), 4.62(\mathrm{~m}, 1 \mathrm{H}), 4.50(\mathrm{dd}, J=$ $11.9,4.6 \mathrm{~Hz}, 1 \mathrm{H}), 4.19(\mathrm{dd}, J=11.9,2.7 \mathrm{~Hz}, 1 \mathrm{H}), 2.59(\mathrm{~m}, 1 \mathrm{H}), 2.43(\mathrm{~s}, 3 \mathrm{H}), 2.24-2.41$ $(\mathrm{m}, 2 \mathrm{H}), 1.97(\mathrm{~m}, 1 \mathrm{H}), 1.91(\mathrm{~s}, 3 \mathrm{H}) ;{ }^{13} \mathrm{C} \mathrm{NMR}\left(125 \mathrm{MHz}, \mathrm{CDCl}_{3}\right) \delta 173.5,170.3,145.2$, 135.6, 129.5 (2C), 128.3 (2C), 65.5, 57.7, 31.0, 22.6, 21.6, 20.6; HRMS (ESI-TOF) calculated for $[\mathrm{M}+\mathrm{H}]^{+} 312.09$, found 312.0908 .

7:

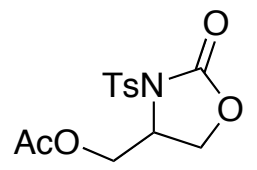

Purified by flash chromatography $\left(4: 4: 1 \rightarrow 2: 2: 1\right.$ hexanes $\left./ \mathrm{CH}_{2} \mathrm{Cl}_{2} / \mathrm{Et}_{2} \mathrm{O}\right)$; TLC: $\mathrm{R}_{f}=0.30$ (3:3:1 hexanes $/ \mathrm{CH}_{2} \mathrm{Cl}_{2} / \mathrm{Et}_{2} \mathrm{O}$ ); IR (film) $1785,1746,1368,1229,1173 \mathrm{~cm}^{-1} ;{ }^{1} \mathrm{H} \mathrm{NMR}$ $\left(400 \mathrm{MHz}, \mathrm{CDCl}_{3}\right) \delta 7.93(\mathrm{~d}, J=8.3 \mathrm{~Hz}, 2 \mathrm{H}), 7.34(\mathrm{~d}, J=8.3 \mathrm{~Hz}, 2 \mathrm{H}), 4.70(\mathrm{~m}, 1 \mathrm{H})$, $4.44(\mathrm{~m}, 2 \mathrm{H}), 4.25(\mathrm{dd}, J=12.0,2.5 \mathrm{~Hz}, 1 \mathrm{H}), 4.21(\mathrm{dd}, J=9.0,3.2 \mathrm{~Hz}, 1 \mathrm{H}), 2.43(\mathrm{~s}, 3 \mathrm{H})$, $1.89(\mathrm{~s}, 3 \mathrm{H}) ;{ }^{13} \mathrm{C}$ NMR $\left(100 \mathrm{MHz}, \mathrm{CDCl}_{3}\right) \delta 170.2,151.9,145.7,134.7,129.7$ (2C), 128.3 (2C), 65.6, 63.4, 55.1, 21.6, 20.3; HRMS (ESI-TOF) calculated for $[\mathrm{M}+\mathrm{Na}]^{+}$ 336.0512 , found 336.0516 .

8:

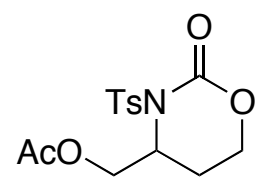

Purified by flash chromatography $\left(4: 4: 1 \rightarrow 2: 2: 1\right.$ hexanes/ $\left.\mathrm{CH}_{2} \mathrm{Cl}_{2} / \mathrm{Et}_{2} \mathrm{O}\right)$; TLC: $\mathrm{R}_{f}=0.18$ (4:4:1 hexanes $/ \mathrm{CH}_{2} \mathrm{Cl}_{2} / \mathrm{Et}_{2} \mathrm{O}$ ); IR (film) 1739, 1353, 1234, $1175,1158 \mathrm{~cm}^{-1} ;{ }^{1} \mathrm{H} \mathrm{NMR}$ $\left(500 \mathrm{MHz} \mathrm{CDCl}_{3}\right) \delta 7.88(\mathrm{~d}, J=8.6 \mathrm{~Hz}, 2 \mathrm{H}), 7.29(\mathrm{~d}, J=7.9 \mathrm{~Hz}, 2 \mathrm{H}), 4.83(\mathrm{~m}, 1 \mathrm{H})$, $4.40(\mathrm{~m}, 2 \mathrm{H}), 4.27(\mathrm{~m}, 2 \mathrm{H}), 2.40(\mathrm{~s}, 3 \mathrm{H}), 2.09-2.25(\mathrm{~m}, 2 \mathrm{H}), 2.02(\mathrm{~s}, 3 \mathrm{H}) ;{ }^{13} \mathrm{C}$ NMR (125 
$\left.\mathrm{MHz}, \mathrm{CDCl}_{3}\right) \delta 170.2,148.3,145.1,135.3,129.3$ (2C), 128.9 (2C), 64.8, 63.5, 52.6, 24.4, 21.5, 20.5; HRMS (ESI-TOF) calculated for [M+Na] 350.0669 , found 350.0682 .

9:

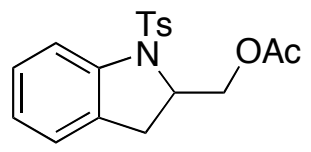

Purified by flash chromatography (3:1 hexanes/ethyl acetate); TLC: $\mathrm{R}_{f}=0.40$ (3:1 hexanes/ethyl acetate); IR (film) 1742, 1598, 1354, 1232, $1168 \mathrm{~cm}^{-1} ;{ }^{1} \mathrm{H}$ NMR $(500 \mathrm{MHz}$, $\left.\mathrm{CDCl}_{3}\right) \delta 7.67(\mathrm{~d}, J=7.9 \mathrm{~Hz}, 1 \mathrm{H}), 7.51(\operatorname{app~d}, J=8.2 \mathrm{~Hz}, 2 \mathrm{H}), 7.22(\mathrm{~m}, 1 \mathrm{H}), 7.15(\mathrm{~d}, J$ $=7.9 \mathrm{~Hz}, 2 \mathrm{H}), 7.04(\mathrm{~d}, J=4.3 \mathrm{~Hz}, 2 \mathrm{H}), 4.51(\mathrm{~m}, 1 \mathrm{H}), 4.17(\mathrm{~m}, 2 \mathrm{H}), 2.76(\mathrm{dd}, J=16.3$, $9.7 \mathrm{~Hz}, 1 \mathrm{H}), 2.57(\mathrm{dd}, J=16.2,2.5 \mathrm{~Hz}, 1 \mathrm{H}), 2.35(\mathrm{~s}, 3 \mathrm{H}), 2.06(\mathrm{~s}, 3 \mathrm{H}) ;{ }^{13} \mathrm{C}$ NMR $(125$ $\left.\mathrm{MHz}, \mathrm{CDCl}_{3}\right) \delta 171.0,144.0,141.2,134.9,131.5,129.6(2 \mathrm{C}), 127.9,127.0$ (2C), 125.0 (2C), 117.8, 65.7, 60.0, 31.4, 21.5, 20.8; HRMS (ESI-TOF) calculated for $[\mathrm{M}+\mathrm{Na}]^{+}$ 368.0927, found 368.0925.

10:

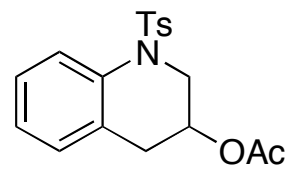

Purified by flash chromatography (3:1 hexanes/ethyl acetate); TLC: $\mathrm{R}_{f}=0.44$ (3:1 hexanes/ethyl acetate); IR (film) 1740, 1490, 1351, 1236, $1165 \mathrm{~cm}^{-1} ;{ }^{1} \mathrm{H}$ NMR $(500 \mathrm{MHz}$, $\left.\mathrm{CDCl}_{3}\right) \delta 7.71(\mathrm{~d}, J=8.2 \mathrm{~Hz}, 1 \mathrm{H}), 7.57(\operatorname{app~d}, J=8.2 \mathrm{~Hz}, 2 \mathrm{H}), 7.21(\mathrm{~m}, 3 \mathrm{H}), 7.10$ (app t, $J=7.4 \mathrm{~Hz}, 1 \mathrm{H}), 7.02(\mathrm{~m}, 1 \mathrm{H}), 4.97(\mathrm{~m}, 1 \mathrm{H}), 4.11(\mathrm{dd}, J=13.9,4.6 \mathrm{~Hz}, 1 \mathrm{H}), 3.73(\mathrm{dd}, J=$ 14.0, $6.8 \mathrm{~Hz}, 1 \mathrm{H}), 2.74(\mathrm{dd}, J=16.5,5.8 \mathrm{~Hz}, 1 \mathrm{H}), 2.54(\mathrm{dd}, J=16.5,6.4 \mathrm{~Hz}, 1 \mathrm{H}), 2.39$ $(\mathrm{s}, 3 \mathrm{H}), 2.01(\mathrm{~s}, 3 \mathrm{H}) ;{ }^{13} \mathrm{C} \mathrm{NMR}\left(125 \mathrm{MHz}, \mathrm{CDCl}_{3}\right) \delta 170.2,143.8,136.9,136.4,129.9$, 129.7 (2C), 129.4, 127.5, 127.1 (2C), 125.5, 124.2, 66.6, 49.1, 32.4, 21.5, 21.0; HRMS (ESI-TOF) calculated for $[\mathrm{M}+\mathrm{Na}]^{+}$368.0927, found 368.0935 .

11ß:

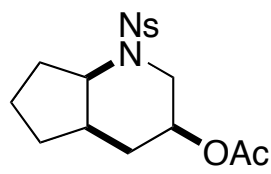

Purified by flash chromatography $\left(8: 8: 1\right.$ hexanes/ $\left.\mathrm{CH}_{2} \mathrm{Cl}_{2} / \mathrm{Et}_{2} \mathrm{O}\right)$; TLC: $\mathrm{R}_{f}=0.44$ (8:8:1 hexanes $/ \mathrm{CH}_{2} \mathrm{Cl}_{2} / \mathrm{Et}_{2} \mathrm{O}$ ); IR (film) 1733, 1544, 1369, 1239, $1163 \mathrm{~cm}^{-1} ;{ }^{1} \mathrm{H}$ NMR (400 MHz, $\left.\mathrm{CDCl}_{3}\right) \delta 8.09(\mathrm{~m}, 1 \mathrm{H}), 7.70(\mathrm{~m}, 2 \mathrm{H}), 7.64(\mathrm{~m}, 1 \mathrm{H}), 4.60(\mathrm{~m}, 1 \mathrm{H}), 4.17(\mathrm{~m}, 1 \mathrm{H}), 3.84(\mathrm{dd}$, $J=12.8,4.9 \mathrm{~Hz}, 1 \mathrm{H}), 2.93(\mathrm{dd}, J=12.8,10.8 \mathrm{~Hz}, 1 \mathrm{H}), 2.19(\mathrm{~m}, 1 \mathrm{H}), 2.03(\mathrm{~s}, 3 \mathrm{H}), 1.84-$ $1.92(\mathrm{~m}, 1 \mathrm{H}), 1.62-1.83(\mathrm{~m}, 5 \mathrm{H}), 1.41(\mathrm{~m}, 1 \mathrm{H}), 1.27(\mathrm{~m}, 1 \mathrm{H}) ;{ }^{13} \mathrm{C}$ NMR $(125 \mathrm{MHz}$, $\left.\mathrm{CDCl}_{3}\right) \delta 169.9,147.8,133.6,133.3,131.8,131.1,124.3,68.3,56.8,43.0,36.1,31.6$, 28.6, 23.4, 21.0, 20.0; HRMS (ESI-TOF) calculated for $[\mathrm{M}+\mathrm{Na}]^{+} 391.0934$, found 391.0941 .

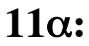

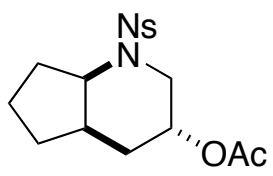


Purified by flash chromatography $\left(8: 8: 1\right.$ hexanes $\left./ \mathrm{CH}_{2} \mathrm{Cl}_{2} / \mathrm{Et}_{2} \mathrm{O}\right)$, inseparable from minor product 12; TLC: $\mathrm{R}_{f}=0.33$ (8:8:1 hexanes/ $\mathrm{CH}_{2} \mathrm{Cl}_{2} / \mathrm{Et}_{2} \mathrm{O}$ ); IR (film) 1737, 1544, 1373, 1242, $1165 \mathrm{~cm}^{-1} ;{ }^{1} \mathrm{H}$ NMR (400 MHz, $\left.\mathrm{CDCl}_{3}\right) \delta 8.08(\mathrm{~m}, 1 \mathrm{H}), 7.67(\mathrm{~m}, 3 \mathrm{H}), 4.87$ (br s, $1 \mathrm{H}), 4.23(\mathrm{~m}, 1 \mathrm{H}), 3.85$ (br d, $J=14.7 \mathrm{~Hz}, 1 \mathrm{H}), 3.20$ (dd, $J=14.7,1.7 \mathrm{~Hz}, 1 \mathrm{H}), 2.39$ (m, $1 \mathrm{H}), 1.83(\mathrm{~s}, 3 \mathrm{H}), 1.58-1.83(\mathrm{~m}, 6 \mathrm{H}), 1.40(\mathrm{~m}, 2 \mathrm{H}) ;{ }^{13} \mathrm{C} \mathrm{NMR}\left(125 \mathrm{MHz}, \mathrm{CDCl}_{3}\right) \delta 170.3$, 147.8, 134.1, 133.2, 131.7, 131.1, 124.3, 66.2, 57.1, 42.8, 31.5, 29.1, 28.4, 23.4, 20.9, 19.9; HRMS (ESI-TOF) calculated for $[\mathrm{M}+\mathrm{Na}]^{+}$391.0934, found 391.0933.

13:

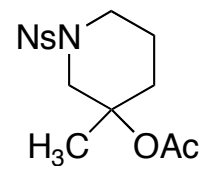

Purified by flash chromatography (8:8:1 hexanes/ $\left.\mathrm{CH}_{2} \mathrm{Cl}_{2} / \mathrm{Et}_{2} \mathrm{O}\right)$; TLC: $\mathrm{R}_{f}=0.35$ (8:8:1 hexanes $/ \mathrm{CH}_{2} \mathrm{Cl}_{2} / \mathrm{Et}_{2} \mathrm{O}$ ); IR (film) 1734, 1544, 1371, 1247, $1164 \mathrm{~cm}^{-1} ;{ }^{1} \mathrm{H}$ NMR $(500 \mathrm{MHz}$, $\left.\mathrm{CDCl}_{3}\right) \delta 8.02(\mathrm{~m}, 1 \mathrm{H}), 7.68(\mathrm{~m}, 2 \mathrm{H}), 7.62(\mathrm{~m}, 1 \mathrm{H}), 4.02(\mathrm{~d}, J=13.1 \mathrm{~Hz}, 1 \mathrm{H}), 3.64$ (br d, $J=12.8 \mathrm{~Hz}, 1 \mathrm{H}), 2.95(\mathrm{~m}, 1 \mathrm{H}), 2.90(\mathrm{~d}, J=13.1 \mathrm{~Hz}, 1 \mathrm{H}), 2.23($ br d, $J=13.7 \mathrm{~Hz}, 1 \mathrm{H})$, $1.91(\mathrm{~s}, 3 \mathrm{H}), 1.83-1.92(\mathrm{~m}, 1 \mathrm{H}), 1.62(\mathrm{~m}, 1 \mathrm{H}), 1.49(\mathrm{~s}, 3 \mathrm{H}), 1.45-1.52(\mathrm{~m}, 1 \mathrm{H}) ;{ }^{13} \mathrm{C} \mathrm{NMR}$ $\left(125 \mathrm{MHz}, \mathrm{CDCl}_{3}\right) \delta 170.3,148.0,133.4,132.7,131.6,131.3,124.0,52.9,45.8,34.5$, 22.6, 22.0, 21.3; HRMS (ESI-TOF) calculated for $[\mathrm{M}+\mathrm{Na}]^{+} 365.0778$, found 365.0778.

15:

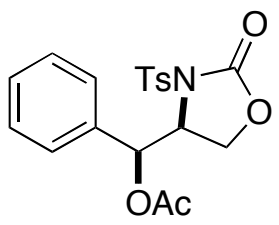

Purified by flash chromatography (4:4:1 hexanes/ $\left.\mathrm{CH}_{2} \mathrm{Cl}_{2} / \mathrm{Et}_{2} \mathrm{O}\right)$; TLC: $\mathrm{R}_{f}=0.53$ (4:4:1 hexanes $/ \mathrm{CH}_{2} \mathrm{Cl}_{2} / \mathrm{Et}_{2} \mathrm{O}$ ); IR (film) 1787, 1751, 1369, 1229, 1172, $1145 \mathrm{~cm}^{-1} ;{ }^{1} \mathrm{H} \mathrm{NMR}$ (500 $\left.\mathrm{MHz} \mathrm{CDCl}_{3}\right) \delta 7.97(\mathrm{~d}, J=8.2 \mathrm{~Hz}, 2 \mathrm{H}), 7.29-7.38(\mathrm{~m}, 7 \mathrm{H}), 6.14(\mathrm{~d}, J=5.2 \mathrm{~Hz}, 1 \mathrm{H})$, $4.81(\mathrm{~m}, 1 \mathrm{H}), 4.30(\mathrm{dd}, J=9.5,2.1 \mathrm{~Hz}, 1 \mathrm{H}), 4.20(\mathrm{dd}, J=9.5,7.9 \mathrm{~Hz}, 1 \mathrm{H}), 2.45(\mathrm{~s}, 3 \mathrm{H})$, $2.14(\mathrm{~s}, 3 \mathrm{H}) ;{ }^{13} \mathrm{C}$ NMR $\left(125 \mathrm{MHz}, \mathrm{CDCl}_{3}\right) \delta 169.1,151.6,145.8,134.8,133.6,129.9$ (2C), 129.1, 128.8 (2C), 128.3 (2C), 126.9 (2C), 73.7, 64.1, 58.7, 21.6, 20.9; HRMS (ESI-TOF) calculated for $[\mathrm{M}+\mathrm{Na}]^{+} 412.0825$, found 412.0828 .

17:

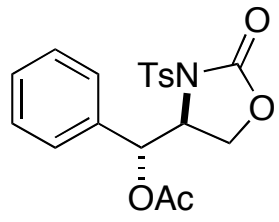

Purified by flash chromatography (4:4:1 hexanes/ $\left.\mathrm{CH}_{2} \mathrm{Cl}_{2} / \mathrm{Et}_{2} \mathrm{O}\right)$; TLC: $\mathrm{R}_{f}=0.48$ (8:8:1 hexanes $/ \mathrm{CH}_{2} \mathrm{Cl}_{2} / \mathrm{Et}_{2} \mathrm{O}$ ); IR (film) 1787, 1754, 1372, 1225, 1172, $1142 \mathrm{~cm}^{-1} ;{ }^{1} \mathrm{H} \mathrm{NMR}$ (500 $\left.\mathrm{MHz} \mathrm{CDCl}_{3}\right) \delta 7.99(\mathrm{~d}, J=8.2 \mathrm{~Hz}, 2 \mathrm{H}), 7.31-7.41(\mathrm{~m}, 5 \mathrm{H}), 7.26(\mathrm{~d}, J=7.0 \mathrm{~Hz}, 2 \mathrm{H})$, 
$6.32(\mathrm{~d}, J=2.1 \mathrm{~Hz}, 1 \mathrm{H}), 4.77(\operatorname{app~dt}, J=8.9,2.4 \mathrm{~Hz}, 1 \mathrm{H}), 4.37(\mathrm{dd}, J=9.2,2.7 \mathrm{~Hz}, 1 \mathrm{H})$, $4.11(\operatorname{app~t}, J=9.2 \mathrm{~Hz}, 1 \mathrm{H}), 2.44(\mathrm{~s}, 3 \mathrm{H}), 1.90(\mathrm{~s}, 3 \mathrm{H}) ;{ }^{13} \mathrm{C} \mathrm{NMR}\left(125 \mathrm{MHz}, \mathrm{CDCl}_{3}\right)$ $\delta 169.1,152.1,145.7,134.7,134.5,129.7$ (2C), 129.1 (2C), 128.8, 128.6 (2C), 125.4 (2C), 73.6, 62.8, 59.7, 21.7, 20.4; HRMS (ESI-TOF) calculated for $[\mathrm{M}+\mathrm{Na}]^{+} 412.0825$, found 412.0822 .

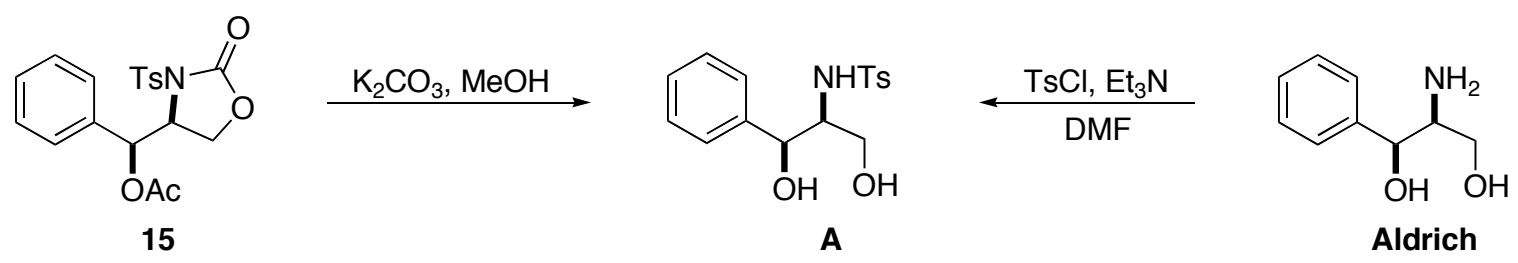

Chemical Correlation for Product 15: For the chemical correlation study, a pure sample of product A was obtained by the $N$-tosylation of $(1 S, 2 S)-(+)-2$-amino-1-phenyl1,3-propanediol (Aldrich) as previously described. ${ }^{6}$ Product $15(28.1 \mathrm{mg}, 0.072 \mathrm{mmol})$ was dissolved in $720 \mu \mathrm{l} \mathrm{MeOH}$, and solid $\mathrm{K}_{2} \mathrm{CO}_{3}(10 \mathrm{mg}, 0.072 \mathrm{mmol})$ was added. The reaction was stirred for $1 \mathrm{~h}$, and quenched with $\mathrm{NH}_{4} \mathrm{Cl}$. The mixture was then extracted with $\mathrm{CHCl}_{3}$ (x 3), and the combined organics were washed with brine and dried $\left(\mathrm{MgSO}_{4}\right)$. Rotary evaporation afforded product A $(20.5 \mathrm{mg}, 89 \%)$ that was identical to the pure sample obtained as above in all respects.

Chemical Correlation Product A: TLC: $\mathrm{R}_{f}=0.61$ (10:1 $\mathrm{CH}_{2} \mathrm{Cl}_{2} /$ methanol); IR (film) 3489, 3289, 1323, 1155, $1092 \mathrm{~cm}^{-1} ;{ }^{1} \mathrm{H}$ NMR (300 MHz, $d^{6}$-DMSO) $\delta 7.45$ (d, $J=8.1$ $\mathrm{Hz}, 2 \mathrm{H}), 7.13-7.26(\mathrm{~m}, 7 \mathrm{H}), 5.37(\mathrm{~d}, J=4.8 \mathrm{~Hz}, 1 \mathrm{H}), 4.88(\mathrm{dd}, J=4.4,3.0 \mathrm{~Hz}, 1 \mathrm{H}), 4.70$ $(\operatorname{app~t}, J=5.4 \mathrm{~Hz}, 1 \mathrm{H}), 3.47(\mathrm{~m}, 1 \mathrm{H}), 3.29(\mathrm{br} \mathrm{s}, 1 \mathrm{H}), 3.15(\mathrm{~m}, 1 \mathrm{H}), 2.32(\mathrm{~s}, 3 \mathrm{H}) ;{ }^{13} \mathrm{C}$ NMR (75 MHz, $d^{6}$-DMSO) $\delta 142.9,141.9,138.9,129.2$ (2C), 127.6 (2C), 126.5, 126.3 (4C), 69.7, 60.8, 60.6, 21.0; HRMS (ESI-TOF) calculated for [M+Na] $]^{+}$344.0927, found 344.0925 .

\section{References:}

1. Kan, T.; Fujiwara, A.; Kobayashi, H.; Fukuyama, T. Tetrahedron 2002, 58, 6267.

2. Fukuyama, T.; Cheung, M.; Kan, T. Synlett 1999, 1301.

3. Pinho, P.; Minnaard, A. J.; Feringa, B. L. Org. Lett. 2003, 5, 259.

4. Larock, R. C.; Pace, P.; Yang, H.; Russell, C. E. Tetrahedron 1998, 54, 9961.

5. For an excellent review discussing the ability of hypervalent iodine reagents to facilitate alkene difunctionalization (in the absence of palladium) see: Zhdankin, V. V.; Stang, P. J. Chem. Rev. 2002, 102, 2523.

6. Righi, P.; Scardovi, N.; Marotta, E.; ten Holte, P.; Zwanenburg, B. Org. Lett. 2002, 4, 497. 